\title{
T-Type Calcium Channels Consolidate Tonic Action Potential Output of Thalamic Neurons to Neocortex
}

\author{
Charlotte Deleuze, ${ }^{1,2}$ François David, ${ }^{2}$ Sébastien Béhuret, ${ }^{1}$ Gérard Sadoc, ${ }^{1}$ Hee-Sup Shin, ${ }^{3}$ Victor N. Uebele, ${ }^{4}$ \\ John J. Renger, ${ }^{4}$ Régis C. Lambert, ${ }^{2 *}$ Nathalie Leresche, ${ }^{2 \star}$ and Thierry Bal ${ }^{1 \star}$ \\ ${ }^{1}$ Unité de Neurosciences, Information et Complexité (UNIC), UPR 3293 CNRS, 91198 Gif-sur-Yvette, France, ${ }^{2}$ Université Pierre et Marie Curie (UPMC), \\ Université Paris 6, UMR7102 CNRS, 75005 Paris, France, ${ }^{3}$ Center for Neural Science, Korea Institute of Science and Technology, Seoul 136-791, Korea, and \\ ${ }^{4}$ Merck Research laboratories, West Point, Pennsylvania 19486
}

The thalamic output during different behavioral states is strictly controlled by the firing modes of thalamocortical neurons. During sleep, their hyperpolarized membrane potential allows activation of the T-type calcium channels, promoting rhythmic highfrequency burst firing that reduces sensory information transfer. In contrast, in the waking state thalamic neurons mostly exhibit action potentials at low frequency (i.e., tonic firing), enabling the reliable transfer of incoming sensory inputs to cortex. Because of their nearly complete inactivation at the depolarized potentials that are experienced during the wake state, T-channels are not believed to modulate tonic action potential discharges. Here, we demonstrate using mice brain slices that activation of T-channels in thalamocortical neurons maintained in the depolarized/wake-like state is critical for the reliable expression of tonic firing, securing their excitability over changes in membrane potential that occur in the depolarized state. Our results establish a novel mechanism for the integration of sensory information by thalamocortical neurons and point to an unexpected role for T-channels in the early stage of information processing.

\section{Introduction}

Nearly all sensory information transmitted to the neocortex, and thus leading to perception, is relayed by the thalamus. During sensory processing, thalamocortical (TC) relay neurons generally exhibit a tonic discharge of spikes, a pattern of activity that strongly contrasts with the rhythmically occurring high-frequency bursts of spikes that are observed during sleep (Llinás and Steriade, 2006). This dichotomic behavior of TC neurons during wakefulness and sleep is currently explained by the properties of the calcium-mediated low-threshold spike (LTS) that underlies the high-frequency bursts (Jahnsen and Llinás, 1984). Indeed, LTSs are generated by T-type calcium channels that are thought to be fully inactivated in the range of membrane potentials associated with the wake state (Coulter et al., 1989; Crunelli et al., 1989) and to require substantial and prolonged hyperpolarizations, such as those present during sleep, to dein-

Received March 19, 2012; revised July 4, 2012; accepted July 12, 2012.

Author contributions: C.D., F.D., R.C.L., N.L., and T.B. designed research; C.D. and F.D. performed research; S.B., G.S., H.-S.S., V.N.U., and J.R.R. contributed unpublished reagents/analytic tools; C.D. and F.D. analyzed data; C.D., F.D., R.C.L., N.L., and T.B. wrote the paper.

This work was supported by CNRS Grant LEA528, Agence Nationale de la Recherche Grants ANR-06-Neuro, ANR-10-BLAN-1402, and ANR-MNMP-2009, Direction Générale de l'Armement, and European Union Grants FP6015879 and FP7-269921. We thank A. Daret and P. Galloux for their technical assistance; $A$. Tscherter for contribution to project design; A. Destexhe for providing models; and P. Lory for support with $\mathrm{Ca}_{\mathrm{v}} 3.1^{-1-}$ mice.

${ }^{*}$ R.C.L., N.L., and T.B. contributed equally to this work.

V.N.U. and J.J.R. are employees of Merck and Co., Inc. (United States) and potentially own stock and/or stock options in the company.

Correspondence should be addressed to Thierry Bal, UNIC, CNRS UPR 3293, 1, Avenue de la Terrasse, Batiment 32-33, 91198 Gif-sur-Yvette, France. E-mail: thierry.ba@@unic.cnrs-gifffr.

DOI:10.1523/JNEUROSCI.1362-12.2012

Copyright $\odot 2012$ the authors $\quad 0270-6474 / 12 / 3212228-09 \$ 15.00 / 0$ activate and generate bursting activity (for review, see Llinás and Steriade, 2006). More recently, sparse bursting preceded by a prolonged period of silence has also been reported in awake animals (Guido and Weyand, 1995; Ramcharan et al., 2000; Fanselow et al., 2001; Swadlow and Gusev, 2001; Martinez-Conde et al., 2002) and attributed to activation of T-channels that deinactivate during the silent period (Llinás and Steriade, 2006; Wang et al., 2007). Therefore, the role of T-channels in thalamic physiology has been restricted to rhythmic bursting during sleep or to occasional isolated bursts during sensory processing, which are always conditioned by a preceding hyperpolarization.

However, the density of T-channels in TC neurons far exceeds that required to generate a LTS following strong hyperpolarization (Bessaïh et al., 2008; Dreyfus et al., 2010). Such a high density of channels results in a physiologically meaningful window T-current (IT) (Dreyfus et al., 2010), demonstrating the presence of a number of deinactivated $\mathrm{T}$-channels in the voltage range (approximately $-60 \mathrm{mV}$ ) associated with the wake state (Llinás and Steriade, 2006). These data led us to investigate the role of IT in regulating synaptic information transfer in the depolarized state. Using the novel highly specific blocker of T-type channels, TTA-P2 (Shipe et al., 2008; Dreyfus et al., 2010), KO mice lacking the T-channel $\mathrm{Ca}_{\mathrm{v}} 3.1$ subunit (Kim et al., 2001) and the dynamicclamp technique, we demonstrate that the activation of T-channels during wake-like states is a major determinant for single and multiple spike occurrence during tonic firing and for the robustness of the thalamocortical transfer of sensory inputs.

\section{Materials and Methods}

In vitro slice preparation. Experimental procedures with animals were performed according to the guidelines of European Council Directive 
86/609/EEC and the European Treaties series no. 123, and with the approval of the ethics committee "Ile-de-France Sud" (05-019).

C57BL/6J wild-type or $\mathrm{Ca}_{\mathrm{v}} 3.1^{-1-}$ (Kim et al., 2001) mice of either sex, 14-23 d old, were anesthetized with inhaled isoflurane and decapitated, and their brain was rapidly removed and immersed in cold "cutting" solution $\left(4^{\circ} \mathrm{C}\right)$ containing the following (in $\mathrm{mm}$ ): $234 \mathrm{su}-$ crose, $2.5 \mathrm{KCl}, 1.25 \mathrm{NaH}_{2} \mathrm{PO}_{4}, 10 \mathrm{MgSO}_{4}, 0.5 \mathrm{CaCl}_{2}, 26 \mathrm{NaHCO}_{3}, 10$ glucose, $\mathrm{pH}$ equilibrated to 7.3 with $95 \% \mathrm{O}_{2} / 5 \% \mathrm{CO}_{2}$. Horizontal slices $(300 \mu \mathrm{m})$ were cut from the somatosensory ventrobasal complex (VB) of the thalamus with a vibratome and then incubated in oxygenated artificial aCSF containing the following (in $\mathrm{mm}$ ): 126 $\mathrm{NaCl}, 2.5 \mathrm{KCl}, 1.25 \mathrm{NaH}_{2} \mathrm{PO}_{4}, 2 \mathrm{MgCl}_{2}, 2 \mathrm{CaCl}_{2}, 26 \mathrm{NaHCO}_{3}$ and 10 glucose, $\mathrm{pH} 7.4$, at $32^{\circ} \mathrm{C}$ for $1 \mathrm{~h}$, and subsequently at room temperature, before being transferred to the recording chamber.

Electrophysiology. Whole-cell patch-clamp recordings of TC neurons located in the VB were performed using a Multiclamp 700B amplifier (Molecular Devices). Acquisition and filter frequencies were set at 20 and $10 \mathrm{kHz}$, respectively. Patch electrodes (tip resistance: 2-3 M $\Omega$ ) were filled with a solution containing the following (in mM): $135 \mathrm{~K}$-gluconate, $0.1 \mathrm{CaCl}_{2}, 5 \mathrm{MgCl}_{2}, 1$ EGTA, 10 HEPES, 4 $\mathrm{Na}-\mathrm{ATP}, 15$ phosphocreatine, and $50 \mathrm{U} / \mathrm{ml}$ creatine phosphokinase, $\mathrm{pH}$ adjusted to 7.3 with $\mathrm{KOH}$ (osmolarity $290 \mathrm{mOsm}$ ). The liquid junction potential $(+10 \mathrm{mV})$ was systematically corrected at the beginning of the recording. All recordings were performed at $32^{\circ} \mathrm{C}$. Access and series resistances were constantly monitored, and data from neurons with a $>20 \%$ change from the initial value were discarded. Recorded TC neurons $(n=77)$ had an input resistance of $204 \pm 10 \mathrm{M} \Omega$ and a resting potential of $-71.7 \pm 0.42 \mathrm{mV}$.

In the absence of current injection, cells presented a quiescent electrical activity with scattered synaptic inputs of weak amplitude occurring randomly in a few cells. Glutamatergic and GABAergic synaptic transmissions were systematically blocked using the ionotropic glutamate receptor blockers DNQX $(10 \mu \mathrm{M})$ and DL-AP5 (100 $\mu \mathrm{M})$ and the $\mathrm{GABA}_{\mathrm{A}}$ receptor antagonist gabazine (SR-95531, $10 \mu \mathrm{M}$ ). When necessary, the blocker of voltage-dependent sodium channels TTX $(1 \mu \mathrm{M})$ was added to the extracellular medium. These drugs were obtained from Tocris Bioscience. T-type calcium channels were blocked using the specific blocker developed by Merck TTA-P2 [3,5-dichloro- $N$-[1(2,2-dimethyl-tetrahydro-pyran-4-ylmethyl)-4-fluoro-piperidinx-4-ylmethyl]-benzamide, $3 \mu \mathrm{M}$ ] (Shipe et al., 2008; Dreyfus et al., 2010).

Dynamic-clamp. Our dynamic-clamp system consists of an Intel Quad-Core computer equipped with an acquisition card (NI PCI-6251 ADC/DAC, M series, National Instruments) connected to the amplifier operating in current-clamp mode. The dynamic-clamp software is based on a custom ADC/DAC (analog-to-digital/digital-to-analog) program used for data acquisition and analysis [Elphy2, developed at Unité de Neurosciences, Information et Complexité (UNIC) by Gérard Sadoc] and interfaced with the NEURON simulator version 6.0 (Hines and Carnevale, 1997). NEURON was modified and recompiled to run under the INtime stack (TenAsys), a kernel driver enabling real-time operation under Microsoft Windows OS. Stimulation protocols were run in real time with the acquisition card at $10 \mathrm{kHz}$.

Thalamocortical neurons receive cortical feedback through direct excitatory inputs from cortex and indirect inhibitory projections from the thalamic reticular nucleus, which generate a synaptic background activity. Although the direct excitation of the thalamic reticular neurons by the corticothalamic feedback suggests that excitatory and inhibitory synaptic noises may be partially correlated in vivo, such correlation has never been characterized so far, and in the absence of experimental data these conductances were considered as uncorrelated in the present model. Therefore, based on pre-established models (Wolfart et al., 2005), this synaptic noise was simulated using excitatory ( $g e$ ) and inhibitory ( $g i)$ fluctuating conductances generated as independent stochastic processes and mimicking the effect of thousands of stochastic glutamate- and GABA-releasing synapses (Destexhe et al., 2001). The total synaptic current, $I_{\text {syn }}=g e(t)(V-$ $\left.E_{e}\right)+g i(t)\left(V-E_{i}\right)$, where $V$ is the membrane potential of the neuron, and $E_{e}$ and $E_{i}$ are the reversal potentials for $g e$ and $g i$ and are set here to 0 and $-85 \mathrm{mV}$, respectively, was injected into TC neurons. Each synaptic conductance was described by a stochastic equation:

$$
d g_{x} / d t=-1 / \tau_{x}\left(g_{x}-g_{x 0}\right)+\left(\sqrt{ }\left[2 \sigma_{x}^{2} / \tau_{x}\right]\right) \xi_{x}(t),
$$

where $g_{x}$ is for $g e$ or $g i, \tau_{x}$ is the correlation time, $\sigma_{x}^{2}$ is the variance of the conductance, and $\xi_{x}(t)$ is a Gaussian noise of zero mean and unit variance.

We reproduced the conductance state observed in thalamus during activated states in vivo (Contreras et al., 1996), by increasing the input conductance of the cell by $50 \%$ via the excitatory and inhibitory synaptic components with a ge/gi ratio of 0.25 (magnitude range: $g e, 0.46-2.8 \mathrm{nS}$; gi, $1.8-11 \mathrm{nS}$ ). The correlation time constants were adjusted to $2.7 \mathrm{~ms}$ $\left(\tau_{e}\right)$ and $10.5 \mathrm{~ms}\left(\tau_{i}\right)$ to match the power spectrum of synaptic conductances resulting from thousand of randomly releasing synapses (Destexhe et al., 2001). The variance of the conductance was set to $75 \%$ of the mean value.

Experimental protocols. Sensory glutamatergic AMPA receptor conductances that evoked fast somatic EPSPs (Turner and Salt, 1998) were modeled according to Destexhe et al. (1998a), as follows:

$$
I \mathrm{AMPA}=g \mathrm{AMPA} r(V-E \mathrm{AMPA})
$$

where gAMPA is the maximal conductance, EAMPA is the reversal potential (set here to $0 \mathrm{mV}$ ), and $r$ is the fraction of the receptors in the open state defined as follows:

$$
\begin{gathered}
d r / d t=\alpha(1-r)-\beta r \\
r_{\infty}=\alpha C_{\max } /\left(\alpha C_{\max }+\beta\right) \\
\tau_{r}=1 /\left(\alpha C_{\max }+\beta\right)
\end{gathered}
$$

with $\alpha=0.94 / \mathrm{ms} \mathrm{mm}, \beta=0.18 / \mathrm{ms} \mathrm{mm}, C_{\max }=0.5 \mathrm{~mm}$. The simulated postsynaptic currents presented a $5 \mathrm{~ms}$ decay time constant that was consistent with the fast kinetics of the sensory-evoked synaptic current in VB neurons (Miyata and Imoto, 2006; Hsu et al., 2010).

The following two types of stimulation patterns were used: (1) AMPA conductances of fixed amplitude (15-50 nS) set to trigger a probability of firing close to 0.5 in the biological neuron were distributed following a Poisson process (mean frequency, $10 \mathrm{~Hz}$ ); and (2) randomized sequences of AMPA conductances with variable peak magnitude (maximal range, 0-90 $\mathrm{nS}$ with $1 \mathrm{nS}$ steps) distributed at a fixed frequency $(2 \mathrm{~Hz})$ to elicit independent inputs.

During these sensory stimulation paradigms, the cortical feedback synaptic bombardment was systematically and continuously simulated in TC neurons and the membrane potential was stabilized at a given target mean value $\left(V_{\text {target }}\right)$. First, the fluctuating voltage excluding sensory responses was averaged within a moving window of $100 \mathrm{~ms}\left(V_{\text {average }}\right)$ and an update current was calculated as follows:

$$
I_{\text {update }}=g\left(V_{\text {average }}-V_{\text {target }}\right),
$$

where $g=200 \mathrm{nS}$. This resulting current was used to perform a weighted update on the injected current such as $I_{\text {injected }}=I_{\text {previous }}+\left(I_{\text {update }}-\right.$ $\left.I_{\text {previous }}\right) / w$, where $I_{\text {previous }}$ was the previously injected current and $w=$ $10^{6}$. This procedure ensured smooth variations of the injected current in response to a slowly changing level of membrane potential.

Data analysis and statistics. The response to Poisson distributed input series was estimated by calculating the mean firing rate (in spikes per second).

The response to $2 \mathrm{~Hz}$ independent inputs was quantified as the probability that a given AMPA input level generates at least one spike within $30 \mathrm{~ms}$ after the onset of the stimulus. The resulting probabilities were used to construct the input-output curve (transfer function) that was fitted to a sigmoidal function: $1-1 /\left(1+\exp \left[\left(x-g \mathrm{AMPA}_{0.5}\right) / d x\right]\right)$, where $g \mathrm{AMPA}_{0.5}$ is the AMPA conductance required to elicit a firing probability of 0.5 .

Single-spike and multispike responses were further distinguished. Responses were considered as multispikes if the interspike interval (ISI) was $<10 \mathrm{~ms}$. Single-spike responses were fitted using a Gaussian function, $A /[w \vee(\pi / 2)] \exp \left[-2\left(x-x_{c}\right)^{2} / \mathrm{w}^{2}\right]$, where $x_{c}$ is the $x$ peak value, $w$ is the width, and $A$ is the area of the Gaussian. 
EPSPs evoked in the presence of TTX by the $2 \mathrm{~Hz}$ independent inputs were averaged to perform the AMPA-triggered average analysis.

Results are reported as the mean \pm SEM. Paired Student's $t$ test was used to compare the data collected with and without T-current or at different voltage levels. Independent Student's $t$ test was used to compare the voltage dependence of data obtained from the Poisson process paradigm in control conditions and after T-current blockade.

Model of intrinsic currents and computational simulations. A singlecompartment conductance model of TC neurons based on Destexhe et al. (1998b) was developed and challenged by sensory gAMPA conductances and $g e$, gi fluctuating noise conductances as in vitro experiments. Thirty-minute-long simulations were typically performed.

The model TC neuron was constructed as follows:

$$
C * d V / d t=-I_{\text {leak }}-I_{\mathrm{T}}-I_{\mathrm{h}}-I_{\mathrm{Na}}-I_{\mathrm{K}},
$$

where $C(0.9 \mathrm{pF})$ is the membrane capacitance, $V$ is the membrane potential of the model neuron, $I_{\text {leak }}$ is the leak current, $I_{\mathrm{T}}$ is the T-type $\mathrm{Ca}^{2+}$ current simulated by a Hodgkin-Huxley-like model fitted to experimental data using empirical functions of voltage (Huguenard and McCormick, 1992; Destexhe et al., 1998b), $I_{\mathrm{h}}$ is the hyperpolarization-activated nonspecific cationic current, $I_{\mathrm{Na}}$ is the voltage-dependent $\mathrm{Na}^{+}$current, and $I_{\mathrm{K}}$ is the delayed rectifier $\mathrm{K}^{+}$current (adapted from Traub and Miles, 1991), to match the spike threshold of the experimentally recorded neurons; each was respectively simulated as follows:

$$
I_{\mathrm{T}}=g_{\mathrm{Ca}} \mathrm{m}^{2} \mathrm{~h}\left(V-E_{\mathrm{Ca}}\right),
$$

where $g_{\mathrm{Ca}}$ is the maximal conductance (set here to $15-125 \mathrm{nS}$ ), $E_{\mathrm{Ca}}$ is the reversal potential for $\mathrm{Ca}^{2+}$ flux (set here to $120 \mathrm{mV}$ ). $m$ and $h$ are activation and inactivation variables, respectively, which are defined as follows:

$$
\begin{aligned}
& d m / d t=\left(m-m_{\infty}(V)\right) / \tau_{m}(V) \\
& \left.d h / d t=\left(h-h_{\infty}(V)\right) / \tau_{h}(V)\right),
\end{aligned}
$$

where

$$
\begin{gathered}
m_{\infty}(V)=1 /(1+\exp [-(V+57) / 6.2]) \\
h_{\infty}(V)=1 /(1+\exp [(V+81) / 4]) \\
\tau_{j}(V)=(0.612+1 /(\exp [-(V+132) / 16.7] \\
+\exp [(V+16.8) / 18.2])) / Q \\
\tau_{h}(V)=(28+\exp [-(V+22) / 10.5]) / Q \text { for } V \geq-81 \mathrm{mV} \\
\tau_{h}(V)=(\exp [(V+467) / 66.6]) / Q \text { for } V<-81 \mathrm{mV} .
\end{gathered}
$$

The variable $Q$ was set to 2.5 corresponding to an experimental temperature of $34^{\circ} \mathrm{C}$ assuming a $Q_{10}$ value of 2.5 with an extracellular $\mathrm{Ca}^{2+}$ concentration of $2 \mathrm{~mm}$ (Coulter et al., 1989).

The same $I_{\mathrm{T}}$ model was used to inject artificial $I_{\mathrm{T}}$ into recorded TC neurons with the dynamic-clamp technique when required. For each neuron, the $g_{\mathrm{Ca}}$ value was adjusted to generate a rebound LTS and associated burst firing that matches the LTS evoked before TTA-P2 application (see example in Fig. 2 A).

$$
\begin{gathered}
I_{h}=g_{h} j\left(V-E_{h}\right) \\
j_{\infty}(V)=1 /(1+\exp [(V+75) / 5.5]) \\
\tau_{j}(V)=1 /(\exp (-14.59-0.086 * V)+\exp (-1.87+0.0701 * V)) \\
d j / d t=\left(j-j_{\infty}(V)\right) / \tau_{j}(V) \\
g_{h}=10 \mathrm{nS}, E_{h}=-33 \mathrm{mV} . \\
I_{\mathrm{Na}}=g_{\mathrm{Na}} \mathrm{m}^{3} \mathrm{~h}\left(V-\mathrm{E}_{\mathrm{Na}}\right) \\
d m / d t=\left(m-m_{\infty}(V)\right) / \tau_{m}(V)
\end{gathered}
$$

$$
\begin{aligned}
& \alpha=1.3 * 0.32 *(13-\nu 2) /(\exp [(13-\nu 2) / 4]-1) \\
& \beta=1.4 * 0.28 *(\nu 2-40) /(\exp [(\nu 2-40) / 5]-1) \\
& \tau_{m}=1 /(\alpha+\beta) / 2 \\
& m_{\infty}=\alpha /(\alpha+\beta) \\
& \left.d h / d t=\left(h-h_{\infty}(V)\right) / \tau_{h}(V)\right) \\
& \alpha=1.3 * 0.128 * \exp [(17-\nu 2) / 18] \\
& \beta=1.3 * 4 /(1+\exp [(40-\nu 2) / 5]) \\
& \tau_{h}=1 /(\alpha+\beta) / 2 \\
& h_{\infty}=\alpha /(\alpha+\beta) \\
& g_{\mathrm{Na}}=13 \mu S, E_{\mathrm{Na}}=50 \mathrm{mV} \text {. } \\
& I_{\mathrm{K}}=g_{\mathrm{K}} \mathrm{n}^{4}\left(V-E_{\mathrm{K}}\right) \\
& d n / d t=\left(n-n_{\infty}(V)\right) / \tau_{n}(V) \\
& \alpha=1.4 * 0.032 *(15-\nu 2) /(\exp [(15-\nu 2) / 5]-1) \\
& \beta=1.6 * 0.5 * \exp [(10-\nu 2) / 40] \\
& \tau_{n}=1 /(\alpha+\beta) / 2 \\
& n_{\infty}=\alpha /(\alpha+\beta) \\
& g_{\mathrm{K}}=420 \mathrm{nS}, E_{\mathrm{K}}=-100 \mathrm{mV} \text {. } \\
& I_{\text {leak }}=g_{\text {leak }}\left(V-E_{\text {leak }}\right) \\
& g_{\text {leak }}=5 \mathrm{nS}, E_{\text {leak }}=-68 \mathrm{mV} \text {. } \\
& \nu 2=V+65
\end{aligned}
$$

The input resistance of the model was set to $200 \mathrm{M} \Omega$ by adjusting the leak conductance.

T-current-dependent responses. In model TC neurons, selection of EPSPs that failed to trigger a spike in the absence of T-conductance $\left(g_{\mathrm{T}}\right)$ (see Fig. 5) was performed as follows. First, a given sequence of $g$ AMPA inputs of various amplitudes and ge/gi noise was run successively in model TC neuron incorporating the $g_{\mathrm{Na}}$ conductance with or without the $g_{\mathrm{T}}$. In these simulations, the $g$ AMPA events that only evoked firing when $g_{\mathrm{T}}$ was present were selected. In a second step, the same simulations were run in model TC neuron devoid of $g_{\mathrm{Na}}$ to isolate the EPSP waveforms and EPSPs generated by the previously selected gAMPA events were averaged.

\section{Results}

Thalamocortical response to sensory input is tuned by background synaptic fluctuations induced by the cortical glutamatergic feedback and the GABAergic intrathalamic activity (Wolfart et al., 2005). In vivo cortical activity depolarizes TC neurons within a $10 \mathrm{mV}$ range of potential, from approximately $-70 \mathrm{mV}$ up to $-60 \mathrm{mV}$ (Dossi et al., 1992). To reproduce in the TC neurons recorded in vitro the noisy synaptic environment of the activated "wake-like" state, we systematically added excitatory and inhibitory fluctuating conductances to the cell membrane, together with sensory-like AMPA conductances using the dynamic-clamp technique. This technique allowed replaying the same sequences of sensory AMPA EPSPs and fluctuating synaptic noise during successive experimental conditions (Wolfart et al., 2005; Destexhe and Bal, 2009). 
A $\mathrm{CTR}$

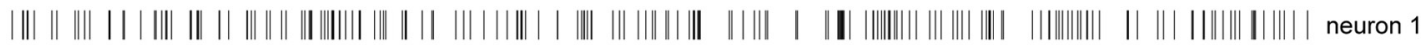
|||||||||||||||||||||||||||||||||||||||||||||||||||||||||||||||||||||||||||||||||||||||||||||||||||||||||||||||||||||||||||||||||||||||||||||||||||||||||||||||||||||||||||||||||||||||||||||||||||||||||||||||||||| neuron 2

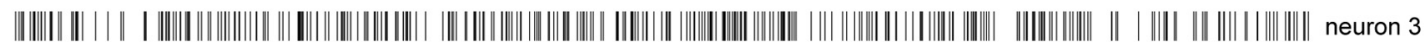
|| | ||| ||| |||||||||||||||||||||||||||||||||||||||||||||||||||||||||||||||||||||||||||||||||||||||||||||||||||||||||||||||||||||||||||||||||||||||||||| neuron 4 |||||||||||||||||||||||||||||||||||||||||||||||||||||||||||||||||||||||||||||||||||||||||||||||||||||||||||||||||||||||||||||||| $\mid$ neuron 5

TTA-P2

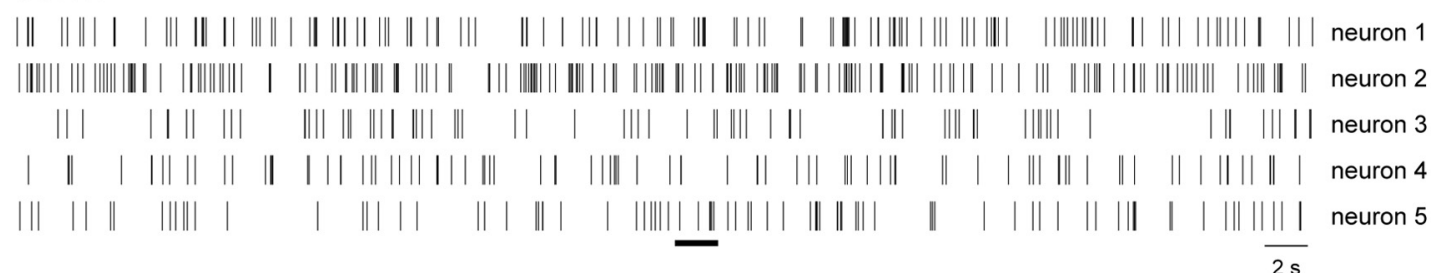

B

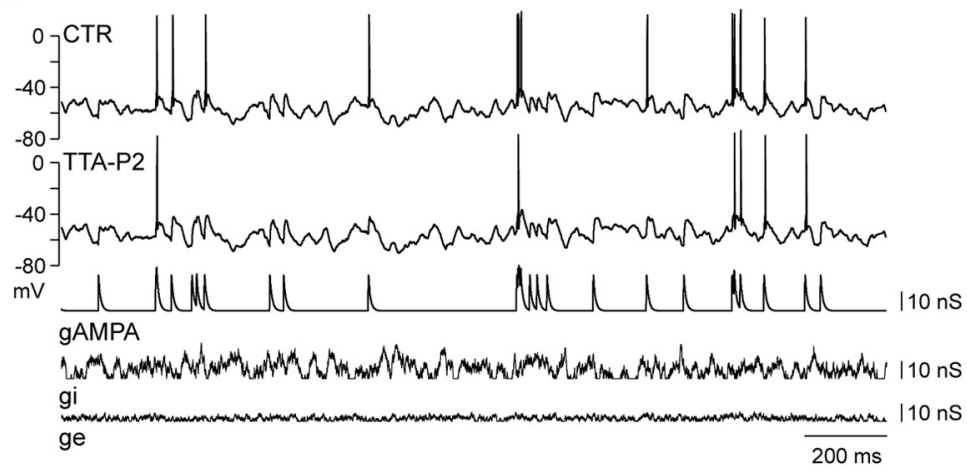

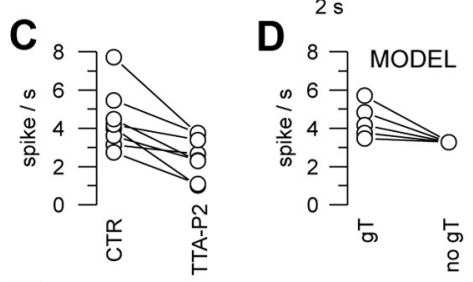

E

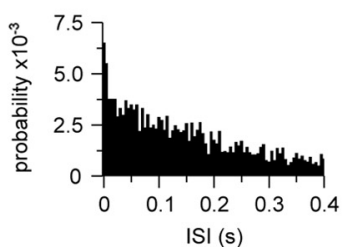

Figure 1. T-current boosts stimulus-evoked firing at depolarized membrane potentials. A, Spike raster plots of five TC neurons injected with gAMPA of fixed amplitude following a Poisson distribution (mean frequency: $10 \mathrm{~Hz}$ ). Neurons were continuously submitted to ge and $g$ i noise, and displayed a mean membrane potential of $-60 \mathrm{mV}$. For each neuron, the same $g$ AMPA/noise sequence was successively injected in the presence [control (CTR)] and absence (TTA-P2) of the T-current. The intracellular activity recorded in neuron 5 during the time windows indicated by the black line in $\boldsymbol{A}$ is illustrated in $\boldsymbol{B}$. The consistent decrease in the number of spikes in TTA-P2 compared with the control condition demonstrates the contribution of T-channels to the firing probability at depolarized potentials. C, Mean firing rates calculated in eight neurons submitted to the same protocol as in $\boldsymbol{A}$. D, Model TC neurons with different T-conductances were submitted to the same protocol as in $A$. The calculated mean firing rates are plotted for each T-conductance values ( $g_{T}$ : left circles, 25, 50, 75, 100, $125 \mathrm{nS}$ from bottom to top; no $g_{\mathrm{T}}$ : right circle) and show that the density of the T-current directly controls the firing probability of TC neurons at depolarized potential. $E$, Cumulative distribution (for the eight neurons presented in C) of ISIs preceding T-current-dependent spikes evoked in the control condition. Spikes were qualified as T-current dependent when they disappeared upon TTA-P2 application. The histogram was normalized to the total area.

T-current increases spike probability in response to sensory synaptic inputs during activated-like states

TC neurons maintained at a depolarized holding potential $(-60.1 \pm 0.1 \mathrm{mV}, n=8)$ were submitted to the same temporal sequence of Poisson-distributed AMPA conductances and synaptic noise in a control condition and in the presence of the specific T-current antagonist, TTA-P2 (Dreyfus et al., 2010). In all recorded neurons, the number of spikes evoked by the AMPA inputs systematically decreased upon T-current blockade (Fig. $1 A-C$ ), inducing a $42.3 \pm 7.3 \%$ reduction in the mean firing rate. We checked that this decrease in the mean firing was not due to a slow run-down of the neuronal activity by performing similar long-term recordings without the application of TTA-P2 in six neurons. In these control experiments, a negligible $1.6 \pm 10.3 \%$ increase in the mean firing rate was observed, confirming the stability of the TC neuron excitability when the T-current was present. Therefore, in contrast to current views, these results clearly demonstrated that T-current plays a significant role in the tonic firing during activated-like states. Moreover, $32 \pm 3 \%$ of the spikes that disappeared upon TTA-P2 application occurred after an interspike interval $<100 \mathrm{~ms}$, contradicting a criterion commonly used in in vivo extracellular recordings (Guido and Weyand, 1995; Ramcharan et al., 2000; Fanselow et al., 2001;
Swadlow and Gusev, 2001; Martinez-Conde et al., 2002) to detect T-current-dependent firing (Fig. $1 E$ ).

A similar protocol applied to model TC neurons with $\mathrm{T}$-conductances of various amplitudes showed that the mean firing rate was positively linked to the magnitude of the T-conductance (Fig. 1D). This result suggests that TC neurons that present a high excitability in the wake-like state express a large T-current and therefore a significant number of deinactivated T-channels at depolarized potentials.

To further characterize the role of the T-current in the transfer of sensory inputs, we submitted TC neurons maintained at a depolarized membrane potential $(-58.2 \pm 0.5 \mathrm{mV}, n=12)$ to simulated AMPA inputs of various amplitudes delivered at a $2 \mathrm{~Hz}$ frequency (Wolfart et al., 2005). As illustrated in Figure 2 B, only the largest AMPA conductances were able to evoke firing when the T-current was antagonized and the transfer function (see Materials and Methods) presented a significant shift toward larger AMPA values (Fig. 2C). We conclude therefore that the T-current not only boosts the response to small AMPA inputs, but increases the firing probability on the whole range of tested AMPA conductances. Quantification performed on the whole TC neuron population indicated that significantly larger AMPA conductances are required to achieve a 0.5 firing probability 
A

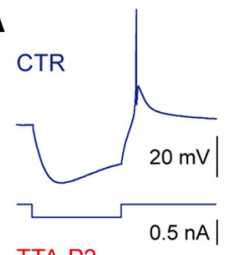

TTA-P2
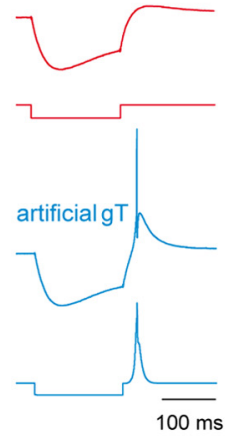

B
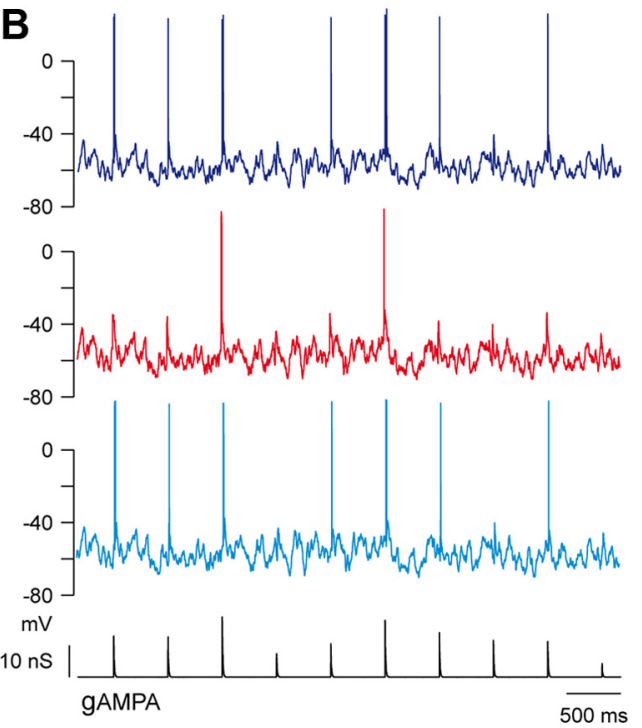

C

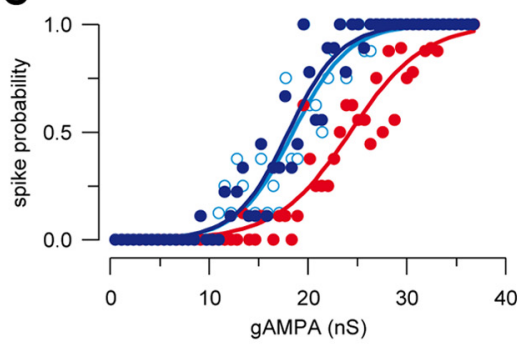

D

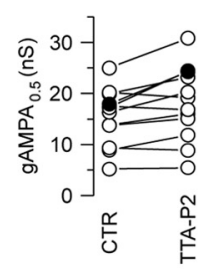

E

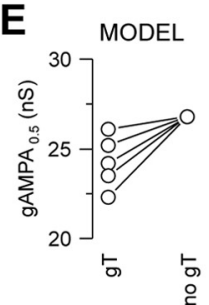

Figure 2. T-current tunes the transfer function of TC neurons at depolarized membrane potentials. $A$, The rebound LTS evoked in a TC neuron following a hyperpolarizing current step (CTR) is blocked by TTA-P2 and restored by injection of $g_{\mathrm{T}} \cdot \boldsymbol{B}$, Voltage traces of a TC neuron injected with a sequence of AMPA conductances ( $g$ AMPA) of different amplitudes in control condition (dark blue) and in the presence of TTA-P2. The neuron received the same fluctuating excitatory and inhibitory conductance noise in each condition and displayed a mean membrane potential of $-58 \mathrm{mV}$. The smallest AMPA conductances failed to evoke a spike when the T-current was blocked (red) and the spike probability was restored upon artificial $g_{\mathrm{T}}$ injection (light blue). $\boldsymbol{C}$, Transfer functions of the neuron presented in $\boldsymbol{B}$ show that the T-current block shifted the input- output curve toward larger AMPA conductances (same color code as in $\boldsymbol{A}$ and $\boldsymbol{B}$ ). Recovery was obtained with injection of $g_{\mathrm{T}}$. $D_{,} g \mathrm{AMPA}_{0.5}$ values were estimated in the same 12 neurons in control condition and in the presence of TTA-P2 (left graph; filled circles correspond to the neuron illustrated in $\boldsymbol{B}$ ). $\boldsymbol{E}$, Model TC neuron: the $\mathrm{gAMPA}_{0.5}$ values decreased with increasing T-conductance amplitudes ( $g_{\mathrm{T}}$ : left circles, $25,50,75,100,125 \mathrm{nS}$ from top to bottom, no $g_{\mathrm{T}}$ : right circles).

$\left(g \mathrm{AMPA}_{0.5}\right)$ in the absence of T-current (Fig. 2D; control: $15.47 \pm 1.60 \mathrm{nS}$, TTA: $18.06 \pm 2.08 \mathrm{nS} ; n=12 ; p<0.01)$. In addition, the number of spikes evoked per input was reduced upon T-current blockade from $1.12 \pm 0.23$ to $0.90 \pm 0.22(n=$ $12 ; p<0.01)$ for the entire range of tested AMPA conductances. The magnitude of the effect was nevertheless variable among TC neurons, but the direction of changes was always consistent and the T-current-dependent shift in the transfer function was clearly present in 8 of the 12 recorded neurons (control: $16.65 \pm 1.68 \mathrm{nS}$; TTA: $20.79 \pm 2.19 \mathrm{nS} ; n=8 ; p<0.001)$. To confirm that this effect specifically depended on T-current, we showed that the shift of the transfer function in the presence of TTA-P2 was reversed by introducing an artificial T-conductance using the dynamic-clamp technique $(n=5$; Fig. $2 A-C)$. Moreover, in computational simulations, $g \mathrm{AMPA}_{0.5}$ values are inversely related to the T-conductance magnitudes (Fig. $2 E$ ), suggesting that the density of T-channels expressed in TC neurons tightly controls the firing probability of sensory AMPA inputs in activatedlike states.

\section{Single-spike and multispike responses at depolarized membrane potential rely on $\mathrm{T}$-current}

As the block of the T-current affected both single-spike and multispike responses (Figs. $2 B, 3 A$ ), we conducted a detailed analysis of the AMPA-induced responses on the eight neurons that demonstrated a clear shift of their transfer function upon block of T-current. The range of AMPA conductances generating singlespike responses was shifted toward larger values upon TTA-P2 application, and the threshold to trigger a single spike became higher (Fig. 3). Moreover, the probability of generating a multispike response drastically decreased or collapsed in the absence of T-current (Fig. $3 B$ ). Therefore, $36 \pm 6 \%$ of the single-spike responses were silenced, a large majority $(79 \pm 8 \%)$ of spike doublets was transformed into single spikes and an equivalent proportion of triplets was transformed either into doublets $(63 \pm$
$7 \%$ ) or single spikes $(19 \pm 6 \%)$. In conclusion, it clearly appears that the T-current controls both the single-spike and multispike responses generated at depolarized membrane potential in response to excitatory inputs.

Moreover, when multispike responses still occurred in the absence of the T-current, their patterns were very similar to the ones evoked in the presence of the T-current with only a slight increase in the first ISI duration (first ISI: $3.72 \pm 0.25 \mathrm{~ms}$ in control vs $4.19 \pm 0.37 \mathrm{~ms}$ in TTA-P2; $n=7 ; p<0.05$; second ISI $3.69 \pm 0.39 \mathrm{~ms}$ in control vs $3.94 \pm 0.49 \mathrm{~ms}$ in TTA-P2; $n=3$ ). Therefore, T-current-dependent multispikes cannot be identified on the basis of their ISI pattern.

\section{T-channel recruitment maintains TC neuron excitability across voltage changes}

Because the T-channel activation and inactivation curves overlap between -70 and $-55 \mathrm{mV}$ (Dreyfus et al., 2010), we hypothesized that the T-current recruitment by sensory inputs should be preserved upon hyperpolarization of the TC neuron in this physiological range of potential and moreover that its impact on the TC response may be enhanced by the increased proportion of deinactivated T-channels. When TC neurons maintained at three different membrane potentials (range: -55 to $-72 \mathrm{mV}$ ) were successively submitted to the same sequence of AMPA inputs of various amplitudes, a large proportion of the small AMPA conductances failed to evoke a spike upon hyperpolarization when T-current was blocked, whereas the spike probability was largely preserved in the control condition (Fig. 4A). Therefore, the transfer function of the TC neurons was quasi-stable across the entire voltage range when T-current was present but shifted toward larger inputs upon hyperpolarization in absence of T-current (Fig. $4 \mathrm{~B}$ ). Interestingly, in TC neurons from $\mathrm{Ca}_{\mathrm{v}} 3.1^{-1-}$ mice that lack endogenous T-channels, a similar shift of the transfer function toward larger AMPA conductances was induced while hyperpolarizing the recorded 
A

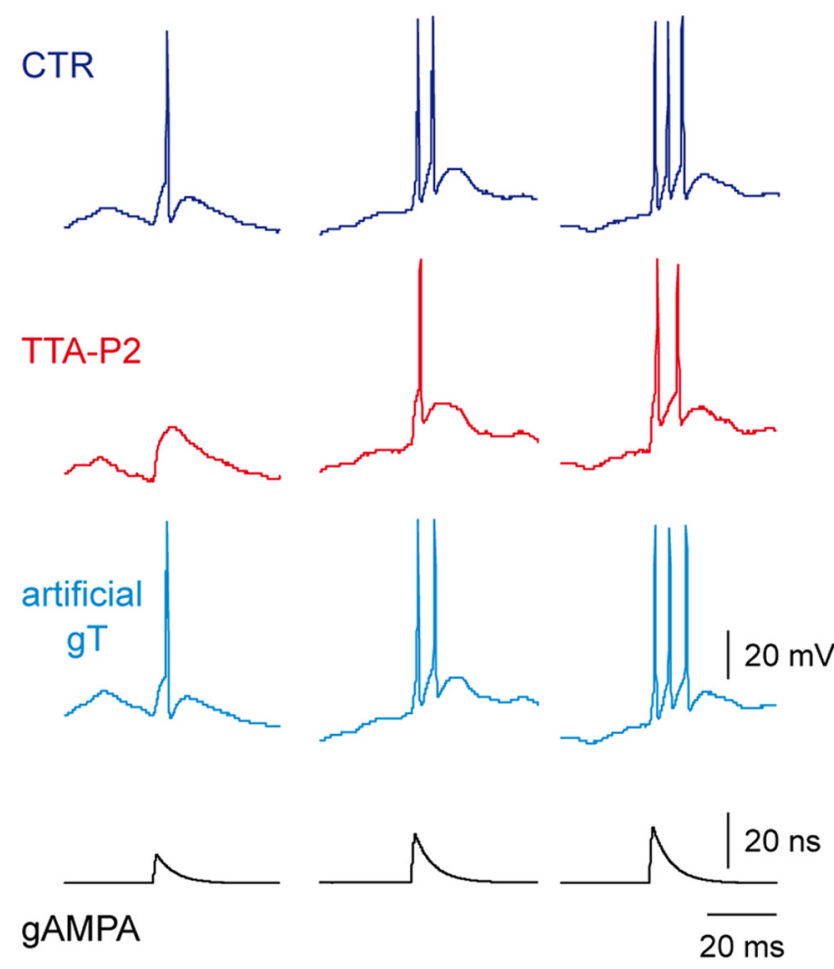

B
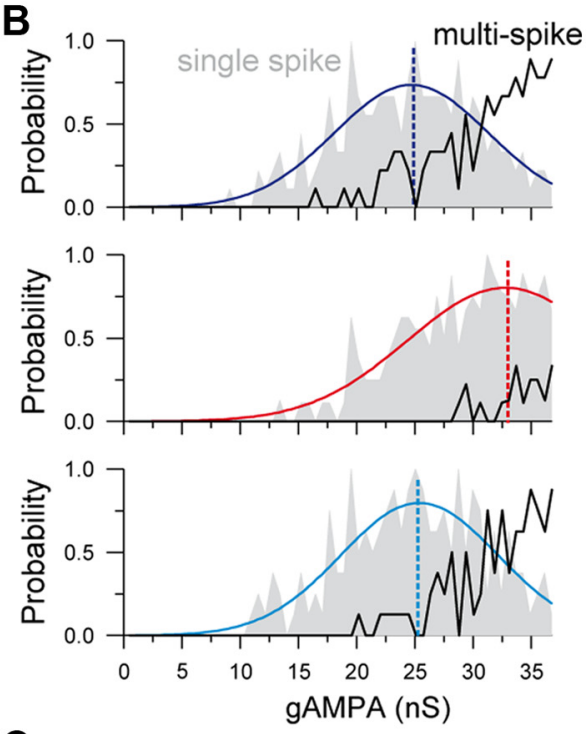

C

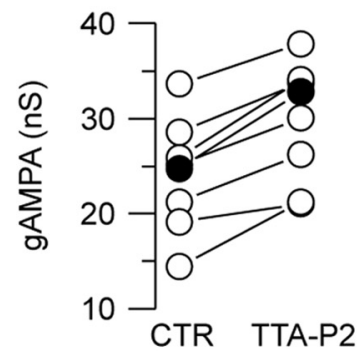

Figure 3. T-current contributes to single-spike and multispike firing. $A$, Examples of firing activity in response to $g$ AMPA of increasing amplitudes recorded in a TC neuron submitted to the same excitatory/inhibitory noise in the control condition (CTR) and in the presence of TTA-P2 (TTA-P2; $g_{\mathrm{T}}$ ), as in Figure $2 B$. Both single-spike and multispike responses were conditioned by the presence of the T-current. Histograms in $\boldsymbol{B}$ present the probability of single-spike (gray area) and multispike (black curve) generation as a function of the $g$ AMPA amplitude in each condition. In the absence of T-current, the single-spike probability curve was shifted toward larger $g$ AMPA and the multispike probability was drastically reduced. The single-spike probability was fitted to a Gaussian function (colored line) to estimate the gAMPA conductance leading to the maximal probability (dashed line). C, The graph presents the gAMPA conductance leading to the maximal single-spike probability calculated in eight neurons in the control condition and in the presence of TTA-P2 (filled circles represent cell in $\boldsymbol{A}$ ).

neurons (Fig. 4C). Quantification performed on the whole TC neuron population (Fig. $4 D$ ) indicated that significantly larger AMPA conductances were required to achieve a 0.5 firing probability at hyperpolarized potentials when the T-current was pharmacologically blocked by TTA-P2 or genetically removed using $\mathrm{Ca}_{\mathrm{v}} 3.1^{-1-}$ mice, but the $g \mathrm{AMPA}_{0.5}$ was either unchanged or even decreased when T-current was present. Numerical simulation showed an inverse relationship between the amplitude of the T-conductance and the magnitude of the shift in the transfer function upon hyperpolarization (Fig. 4E).

Thus, T-current not only contributes to the tonic firing in TC neurons but, due to its graded deinactivation with increasing hyperpolarization, also stabilizes the excitability of the neuronal population across a range of membrane voltages spanned by TC neurons during the waking state.

\section{T-current enhances EPSPs}

To understand how T-current tunes the transfer function of TC neurons at depolarized potential and stabilizes it throughout voltage changes, we analyzed the T-current contribution to EPSP evoked by artificial AMPA conductances in the presence of TTX to block spike generation. At hyperpolarized potentials, the averaged EPSP waveforms were clearly enhanced by the presence of T-current $(-70 \mathrm{mV}$ : amplitude control $27.4 \pm 1.5 \mathrm{mV}$, TTA-P2 $22.2 \pm 1.4 \mathrm{mV}, p<0.01$; half-width control $27.6 \pm 1.9 \mathrm{~ms}$,
TTA-P2 $20.6 \pm 2.3 \mathrm{~ms}, p<0.001 ;-65 \mathrm{mV}$ : amplitude control $22.8 \pm 1.7 \mathrm{mV}$, TTA-P2 $20.1 \pm 1 \mathrm{mV} p<0.05$; half-width control $24.7 \pm 1.8$ ms, TTA-P2 $20.8 \pm 1.8$ ms, $p<0.05 ; n=8$; Fig. $5 A$ ), but an increase in EPSP duration was observed in only two of the six recorded neurons at $-60 \mathrm{mV}$ (Fig. 5A, inset). However, since at depolarized potentials many AMPA inputs are large enough to trigger a spike independently of the T-current, the T-current effect on the averaged EPSP waveform is underestimated when pooling the whole EPSP population. This was confirmed by numerical simulations successively performed in the presence and absence of the sodium and $\mathrm{T}$-conductances that allowed isolation of the EPSPs that required the presence of T-current to generate a spike (see Materials and Methods). As shown in Figure $5 B$, when considering this selected EPSP population (Fig. $5 B 2$, IT-dependent responses), the T-current effects on both the duration and the amplitude of the EPSPs were greatly enhanced and became evident even at the most depolarized potential. We conclude that the impact of T-current on the transfer function involves a graded enhancement of the amplitude and duration of the EPSPs with hyperpolarization.

\section{Discussion}

Our results establish the crucial role played by the T-current in the tonic discharge of TC neurons, a firing mode encoding sensory information transfer to the neocortex during wakefulness, and therefore challenge the classical view that restricts T-current 
A

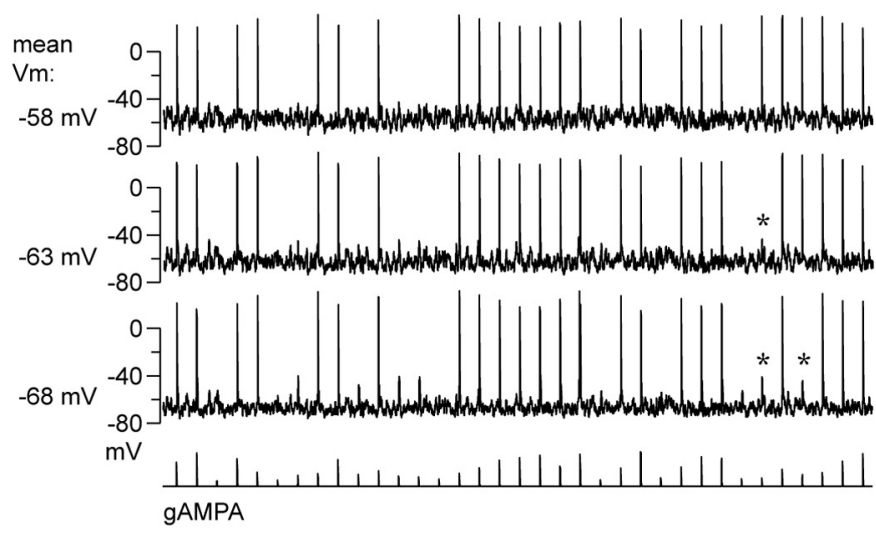

TTA-P2

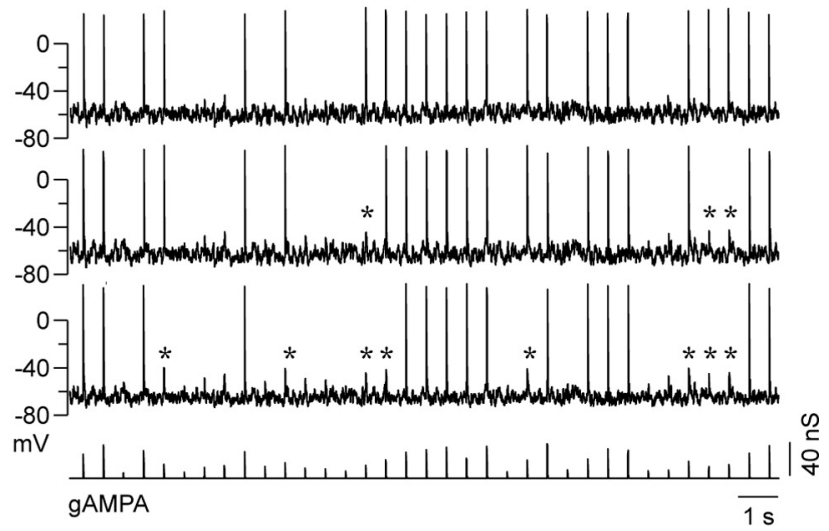

C
B

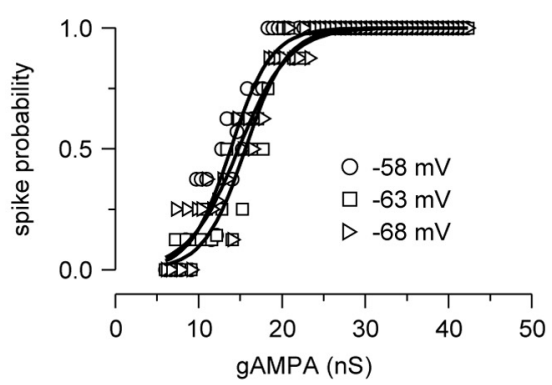

D

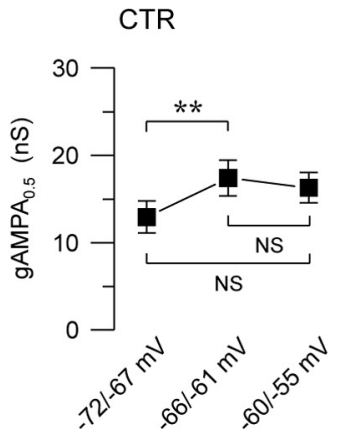

TTA-P2

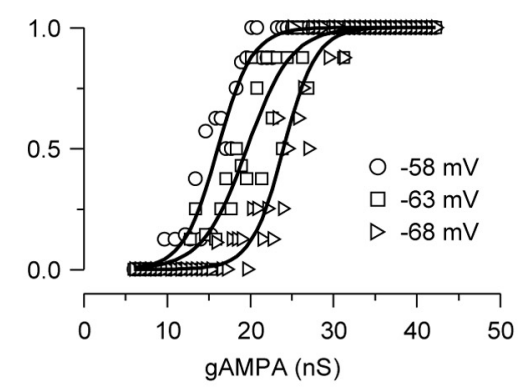

TTA-P2

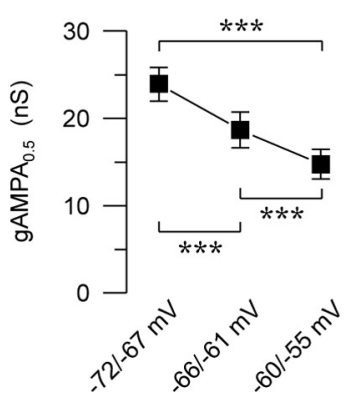

Cav3.1 $1^{-1}$

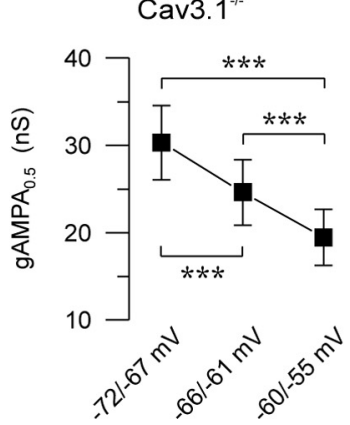

$\mathrm{Ca}_{\mathrm{v}} 3.1^{-1-}$

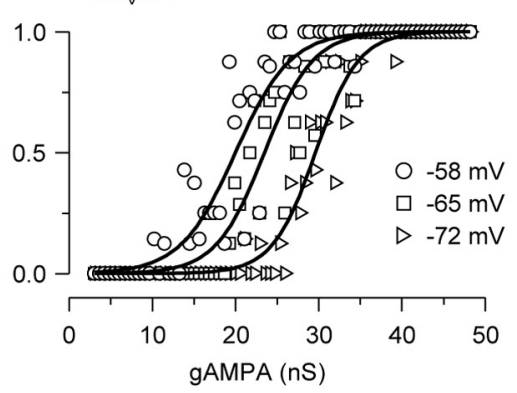

E

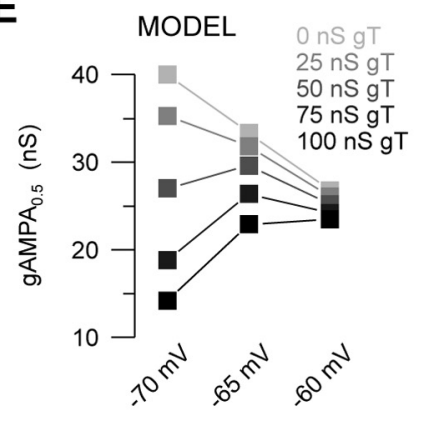

Figure 4. T-current confers robustness to the neuronal response and stabilizes the transfer function of TC neurons across a large range of membrane potentials. $A$, Voltage traces recorded in a TC neuron maintained at mean membrane potentials of $-58,-63$, and $-68 \mathrm{mV}$ while being submitted to AMPA conductances ( $g A M P A)$ of different amplitudes. The same sequences of $g$ AMPA and excitatory/inhibitory noise were injected in every condition. As indicated by asterisks, which point to the loss of spike upon hyperpolarization, the firing probability was barely affected in the control condition (CTR) while it was highly sensitive to membrane potential changes in the presence of TTA-P2. B, Transfer functions were quasi-invariant in the presence of the T-current but drastically shifted toward larger $g$ AMPA values upon hyperpolarization when the T-current was blocked. $C$, Similar voltage dependence of the transfer functions was observed in TC neurons recorded in $\mathrm{Ca}_{\mathrm{v}} 3.1^{-1-}$ knock-out mice devoid of T-current. $\boldsymbol{D}$, Mean values of $g \mathrm{AMPA}_{0.5}$ were determined in TC neurons successively maintained between -72 and $-67 \mathrm{mV},-66 \mathrm{and}-61 \mathrm{mV}$, and -60 and $-55 \mathrm{mV}$. In the control condition (CTR, $n=16), g \mathrm{AMPA}_{0.5}$ values remained stable when the neurons were hyperpolarized from $-60 /-55$ to $-66 /-61 \mathrm{mV}$ and decreased at a more hyperpolarized potential. Conversely, when the T-current was blocked (TTA-P2, $n=12$ ) or absent $\left(\mathrm{Ca}_{\mathrm{v}} 3.1^{-/-}, n=9\right)$, the required AMPA inputs drastically increased with hyperpolarization of the TCneurons (paired $t$ test; NS, nonsignificant; ${ }^{* *} p<0.01 ; * * * 00.001$ ). Note that a subset of cells was recorded at different membrane potentials in only one condition, either CTR ( $n=8$ ) or TTA-P2 $(n=4)$, and that absolute mean values cannot be quantitatively compared across graphs in $\mathbf{D}$. E, TC neuron model: $g \mathrm{AMPA}_{0.5}$ calculated at different membrane voltages for various T-conductances $\left(g_{\mathrm{T}}\right)$. Note the stability conferred by the increasing $g_{\mathrm{T}}$.

recruitment only to the generation of LTS-mediated burst firing. Using TTA-P2, a potent and specific antagonist of T-type calcium channels (Shipe et al., 2008; Dreyfus et al., 2010), and the dynamic-clamp technique, which allows replaying the same fluctuating conductance sequences (Wolfart et al., 2005; Destexhe and Bal, 2009) during successive experimental conditions, we demonstrate that T-current is a major determinant of singlespike and multispike generation at membrane potentials where T-channels were previously considered to be fully inactivated. A key physiological significance of these results is that the increased recruitment of T-channels with hyperpolarization during tonic firing confers a remarkable robustness to the input-output transfer function of TC neurons across a wide voltage range.

T-channel activation at depolarized membrane potentials The exclusive association between T-current- and LTS-mediated burst firing conditioned by strong preceding hyperpolarization (Llinás and Steriade, 2006) emerged from the extensive in vitro characterization of the T-channel properties. These studies established the almost complete inactivation of the T-channels (Coulter et al., 1989; Crunelli et al., 1989) at potentials associated with the wake state (approximately $-60 \mathrm{mV}$ ) (Llinás and Steriade, 
A
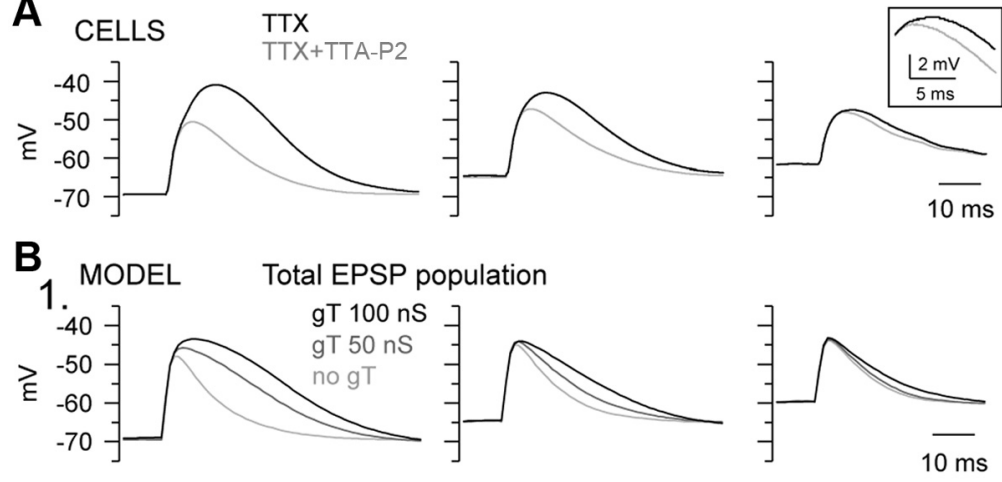

2.

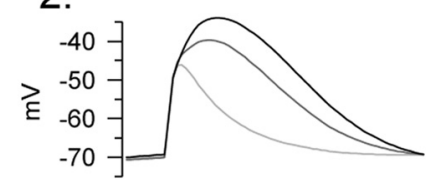

IT dependent responses
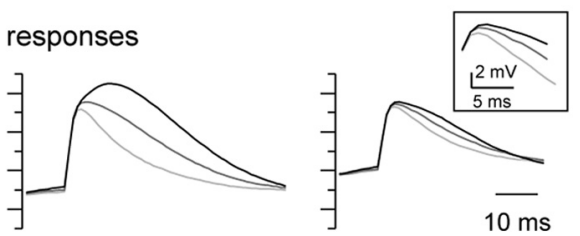

Figure 5. Contribution of the T-current to the EPSP in TC neurons. A, Averaged EPSPs evoked in a TC neuron injected with $g$ AMPA of various amplitudes while being maintained at three different mean membrane potentials in presence of TTX. The same sequences of $g$ AMPA and ge/gi noise were used for each membrane potential in control condition (TTX) and in the presence of TTA-P2 (TTX + TTA-P2). T-current largely contributed to the responses evoked at -70 and $-65 \mathrm{mV}$, and slightly prolonged the average EPSPs recorded at depolarized membrane potential (see inset). $\boldsymbol{B} 1$, Average EPSPs simulated in TC neuron model devoid of $\mathrm{Na}^{+}$current and presenting various $g_{\mathrm{T}}$ values. B2, Selecting EPSPs that failed to trigger spike in the absence of $g_{\mathrm{T}}$ when the $\mathrm{Na}^{+}$ conductance is reintroduced (IT-dependent responses; see Materials and Methods) revealed a prominent contribution of the T-current at every membrane potential (see inset).

2006). However, the density of T-channels expressed in thalamic neurons is especially high, and the tiny fraction of T-channels that are deinactivated at depolarized potentials is physiologically meaningful. Indeed, we previously demonstrated that although the open probability of the channels is low at $-60 \mathrm{mV}$, the number of available channels is sufficient to generate a window current that determines the resting membrane potential of the TC neurons (Dreyfus et al., 2010). Here, we show that the drastic increase in open probability induced by the depolarization associated with sensory AMPA inputs further recruits these deinactivated channels, resulting in a transient T-current that boosts the EPSP and increases the firing probability. Moreover, the amplitude of the current generated by this small population of deinactivated T-channels is enhanced by a voltage-dependent phosphorylation process that specifically occurs at depolarized potentials (Leresche et al., 2004; Bessain et al., 2008). Interestingly, such potentiation of the $\mathrm{T}$-current is only present in TC neurons of the sensory relay nuclei (Leresche et al., 2004), suggesting that the transfer of the sensory information to the cortex requires a non-negligible $\mathrm{T}$-current at depolarized potentials in TC neurons.

\section{Significance for single-spike and multispike recordings in vivo}

A growing number of in vivo studies have reported occasional highfrequency bursts of spikes in sensory thalamic nuclei of awake animals, already suggesting an involvement of the T-current in the transfer of the sensory information (Guido and Weyand, 1995; Ramcharan et al., 2000; Fanselow et al., 2001; Swadlow and Gusev, 2001; Weyand et al., 2001; Martinez-Conde et al., 2002). However, in these studies the contribution of the T-current is restricted to the generation of high-frequency bursts and requires a relatively long preceding hyperpolarization as their authors only analyzed multispike firing preceded by a $100 \mathrm{~ms}$ silent period. In contrast, we clearly show that when the membrane po- tential of the TC neurons randomly fluctuates around a mean value of -55 or $-60 \mathrm{mV}$, the T-current is efficiently recruited by synaptic inputs and boosts the amplitude and duration of the EPSPs facilitating the generation of both singlespike and multispike responses. Thus, the role of the T-current in TC neurons cannot be restricted to the generation of highfrequency bursts. In addition, $>30 \%$ of T-current-dependent spikes followed the preceding spike by $<100 \mathrm{~ms}$, clearly demonstrating that a long silent period is not a prerequisite for the T-current contribution to TC neuron output at depolarized potentials. Moreover, the multispike responses that were dependent on the T-current were temporally indistinguishable from the doublets or triplets of spikes that persisted in its absence. Therefore, standard timing criteria do not accurately separate $\mathrm{T}$-current-mediated from $\mathrm{T}$ current-independent multispike responses and are not a suitable marker for the involvement of the T-current in TC neuron firing.

\section{Voltage invariance of the spike response}

Because activation of the T-current and the subsequent boosting of the depolarizing drive of EPSPs become gradually more prominent with hyperpolarization, the range of membrane potentials where synaptic potentials can reach the spike threshold increases. This accounts for the robustness of the spike response across a large range of voltages, and this newly described intrinsic property impacts on the whole transfer function of the TC neurons that become quasi-invariant to significant membrane potential changes in the region between -55 and $-72 \mathrm{mV}$. Such voltage invariance of EPSPs spike probability may be particularly important in the somatosensory system where the information transfer is believed to rely on a precise spike timing code both at the level of the trigeminal complex (Jones et al., 2004) and the ventroposterior medial nucleus of the thalamus (Montemurro et al., 2007; Petersen et al., 2008). Similarly in the visual system, such a voltage invariance may support the experimentally observed "relative precision" of the temporal structure in the thalamic neuronal response, which is necessary to represent accurately the more slowly changing visual world (Butts et al., 2007; Desbordes et al., 2008).

\section{Significance for sensory processing}

Finally, due to the convergence of multiple sensory inputs on a single TC neuron (Brecht and Sakmann, 2002; Deschênes et al., 2003) and because of the strong short-term plasticity at lemniscal synapses (Castro-Alamancos, 2002), whisker deflection reliably evokes EPSPs with little difference in kinetics but large variability in amplitude. Similar properties have been reported for the retinogeniculate synapses (Turner and Salt, 1998; Chen and Regehr, 2000) where synchronization of the retinal inputs and summation of the postsynaptic potentials (Usrey et al., 1998; Weyand, 2007) enhance the firing probability of TC neurons in the visual thalamus. As a consequence, the sensory code at the level of the TC neuron may partly depend on differences in EPSP amplitude 
and summation. Therefore, the input-output gain function of TC neurons should drastically condition the translation of this sensory code based on the EPSP amplitude variability into different firing patterns of single spikes and multispikes. Accordingly, by closely controlling the input-output gain function of TC neurons, the combination of T-current and synaptic background activity within the thalamocortical loop (Wolfart et al., 2005) represents a major determinant of the sensory information that reaches the primary sensory cortex.

\section{References}

Bessaï T, Leresche N, Lambert RC (2008) T current potentiation increases the occurrence and temporal fidelity of synaptically evoked burst firing in sensory thalamic neurons. Proc Natl Acad Sci U S A 105:11376-11381.

Brecht M, Sakmann B (2002) Whisker maps of neuronal subclasses of the rat ventral posterior medial thalamus, identified by whole-cell voltage recording and morphological reconstruction. J Physiol 538:495-515.

Butts DA, Weng C, Jin J, Yeh CI, Lesica NA, Alonso JM, Stanley GB (2007) Temporal precision in the neural code and the timescales of natural vision. Nature 449:92-95.

Castro-Alamancos MA (2002) Properties of primary sensory (lemniscal) synapses in the ventrobasal thalamus and the relay of high-frequency sensory inputs. J Neurophysiol 87:946-953.

Chen C, Regehr WG (2000) Developmental remodeling of the retinogeniculate synapse. Neuron 28:955-966.

Contreras D, Timofeev I, Steriade M (1996) Mechanisms of long-lasting hyperpolarizations underlying slow sleep oscillations in cat corticothalamic networks. J Physiol 494:251-264.

Coulter DA, Huguenard JR, Prince DA (1989) Calcium currents in rat thalamocortical relay neurones: kinetic properties of the transient, lowthreshold current. J Physiol 414:587-604.

Crunelli V, Lightowler S, Pollard CE (1989) A T-type Ca2 + current underlies low-threshold $\mathrm{Ca} 2+$ potentials in cells of the cat and rat lateral geniculate nucleus. J Physiol 413:543-561.

Desbordes G, Jin J, Weng C, Lesica NA, Stanley GB, Alonso JM (2008) Timing precision in population coding of natural scenes in the early visual system. PLoS Biol 6:e324.

Deschênes M, Timofeeva E, Lavallée P (2003) The relay of high-frequency sensory signals in the whisker-to-barreloid pathway. J Neurosci 23:6778-6787.

Destexhe A, Bal T (2009) Dynamic-clamp: from principles to applications. New York: Springer.

Destexhe A, Mainen ZF, Sejnowski TJ (1998a) Methods in neuronal modeling, Ed 2. Cambridge, MA: MIT.

Destexhe A, Neubig M, Ulrich D, Huguenard J (1998b) Dendritic lowthreshold calcium currents in thalamic relay cells. J Neurosci 18:3574-3588.

Destexhe A, Rudolph M, Fellous JM, Sejnowski TJ (2001) Fluctuating synaptic conductances recreate in vivo-like activity in neocortical neurons. Neuroscience 107:13-24.

Dossi RC, Nuñez A, Steriade M (1992) Electrophysiology of a slow (0.5-4 $\mathrm{Hz}$ ) intrinsic oscillation of cat thalamocortical neurones in vivo. J Physiol 447:215-234.

Dreyfus FM, Tscherter A, Errington AC, Renger JJ, Shin HS, Uebele VN, Crunelli V, Lambert RC, Leresche N (2010) Selective T-type calcium channel block in thalamic neurons reveals channel redundancy and physiological impact of $\mathrm{I}_{\text {Twindow. }}$ J Neurosci 30:99-109.

Fanselow EE, Sameshima K, Baccala LA, Nicolelis MA (2001) Thalamic bursting in rats during different awake behavioral states. Proc Natl Acad Sci U S A 98:15330-15335.

Guido W, Weyand T (1995) Burst responses in thalamic relay cells of the awake behaving cat. J Neurophysiol 74:1782-1786.

Hines ML, Carnevale NT (1997) The NEURON simulation environment. Neural Comput 9:1179-1209.
Hsu CL, Yang HW, Yen CT, Min MY (2010) Comparison of synaptic transmission and plasticity between sensory and cortical synapses on relay neurons in the ventrobasal nucleus of the rat thalamus. J Physiol 588:4347-4363.

Huguenard JR, McCormick DA (1992) Simulation of the currents involved in rhythmic oscillations in thalamic relay neurons. J Neurophysiol 68:1373-1383.

Jahnsen H, Llinás R (1984) Electrophysiological properties of guinea-pig thalamic neurones: an in vitro study. J Physiol 349:205-226.

Jones LM, Depireux DA, Simons DJ, Keller A (2004) Robust temporal coding in the trigeminal system. Science 304:1986-1989.

Kim D, Song I, Keum S, Lee T, Jeong MJ, Kim SS, McEnery MW, Shin HS (2001) Lack of the burst firing of thalamocortical relay neurons and resistance to absence seizures in mice lacking alpha(1G) T-type $\mathrm{Ca}(2+)$ channels. Neuron 31:35-45.

Leresche N, Hering J, Lambert RC (2004) Paradoxical potentiation of neuronal T-type $\mathrm{Ca}^{2+}$ current by ATP at resting membrane potential. J Neurosci 24:5592-5602.

Llinás RR, Steriade M (2006) Bursting of thalamic neurons and states of vigilance. J Neurophysiol 95:3297-3308.

Martinez-Conde S, Macknik SL, Hubel DH (2002) The function of bursts of spikes during visual fixation in the awake primate lateral geniculate nucleus and primary visual cortex. Proc Natl Acad Sci U S A 99:13920-13925.

Miyata M, Imoto K (2006) Different composition of glutamate receptors in corticothalamic and lemniscal synaptic responses and their roles in the firing responses of ventrobasal thalamic neurons in juvenile mice. J Physiol 575:161-174.

Montemurro MA, Panzeri S, Maravall M, Alenda A, Bale MR, Brambilla M, Petersen RS (2007) Role of precise spike timing in coding of dynamic vibrissa stimuli in somatosensory thalamus. J Neurophysiol 98:1871-1882.

Petersen RS, Brambilla M, Bale MR, Alenda A, Panzeri S, Montemurro MA, Maravall M (2008) Diverse and temporally precise kinetic feature selectivity in the VPm thalamic nucleus. Neuron 60:890-903.

Ramcharan EJ, Gnadt JW, Sherman SM (2000) Burst and tonic firing in thalamic cells of unanesthetized, behaving monkeys. Vis Neurosci 17:55-62.

Shipe WD, Barrow JC, Yang ZQ, Lindsley CW, Yang FV, Schlegel KA, Shu Y, Rittle KE, Bock MG, Hartman GD, Tang C, Ballard JE, Kuo Y, Adarayan ED, Prueksaritanont T, Zrada MM, Uebele VN, Nuss CE, Connolly TM, Doran SM, et al (2008) Design, synthesis, and evaluation of a novel 4-aminomethyl-4-fluoropiperidine as a T-type Ca2 + channel antagonist. J Med Chem 51:3692-3695.

Swadlow HA, Gusev AG (2001) The impact of "bursting" thalamic impulses at a neocortical synapse. Nat Neurosci 4:402-408.

Traub RD, Miles R (1991) Multiple modes of neuronal population activity emerge after modifying specific synapses in a model of the CA3 region of the hippocampus. Ann N Y Acad Sci 627:277-290.

Turner JP, Salt TE (1998) Characterization of sensory and corticothalamic excitatory inputs to rat thalamocortical neurones in vitro. J Physiol 510:829-843.

Usrey WM, Reppas JB, Reid RC (1998) Paired-spike interactions and synaptic efficacy of retinal inputs to the thalamus. Nature 395:384-387.

Wang X, Wei Y, Vaingankar V, Wang Q, Koepsell K, Sommer FT, Hirsch JA (2007) Feedforward excitation and inhibition evoke dual modes of firing in the cat's visual thalamus during naturalistic viewing. Neuron 55:465-478.

Weyand TG (2007) Retinogeniculate transmission in wakefulness. J Neurophysiol 98:769-785.

Weyand TG, Boudreaux M, Guido W (2001) Burst and tonic response modes in thalamic neurons during sleep and wakefulness. J Neurophysiol 85:1107-1118.

Wolfart J, Debay D, Le Masson G, Destexhe A, Bal T (2005) Synaptic background activity controls spike transfer from thalamus to cortex. Nat Neurosci 8:1760-1767. 